\title{
A BI-FIDELITY DG-IMEX METHOD FOR THE LINEAR TRANSPORT EQUATION WITH RANDOM PARAMETERS
}

\author{
Liu Liu ${ }^{1}$ \\ ${ }^{1}$ Department of Mathematics, The Chinese University of Hong Kong, \\ Lady Shaw Building, Shatin, N.T., Hong Kong SAR \\ lliu@math.cuhk.edu.hk
}

Key words: linear transport equation, uncertainty quantification, stochastic collocation, bi-fidelity, DG-IMEX

\begin{abstract}
In this paper, we introduce a bi-fidelity stochastic collocation (SC) method for the linear transport equation with diffusive scaling and high-dimensional random inputs characterized by random variables. For the high-fidelity linear transport model, the asymptotic-preserving Discontinuous Galerkin implicit-explicit method in the micro-macro decomposition framework is used. We discuss different choices of low-fidelity models and conduct the corresponding uniform error estimates for the bi-fidelity method. Extensive numerical tests are presented to validate our bi-fidelity SC method.
\end{abstract}

\section{Introduction}

Kinetic equations are widely used in classical fields such as rarefied gas, plasma physics, astrophysics, also in emerging areas such as semiconductor device modeling, social and biological sciences. They model the nonequilibrium dynamics of a large number of particles from a statistical point of view [7]. The solutions to kinetic models determine the probability density functions of the dynamical states of a large number of particles, which usually depend on time, space and velocity.

Uncertainty Quantification (UQ) has drawn many attentions over the past decade. Quantifying uncertainties in the inputs of models is important to assess, validate and improve the underlying models. Kinetic equations, usually derived from $N$-body Newton's equations via the mean field limit [5,6], typically contain integral operators modeling interactions between particles or with the background. The collision kernel or scattering cross-section in this integral describes the transition rate during particle collisions. For complex practical problems, usually only empirical formulas for collision kernels are used. This is our motivation of studying kinetic problems with uncertain inputs, such as random initial or boundary data and random cross-sections.

In UQ, one widely used method is the non-intrusive stochastic collocation (SC) method. There have been many works developed, for example [1,4,15,17,25,26,31]. For many complex systems, an accurate deterministic solver can be really time-consuming and memory demanding, which makes accurate stochastic simulations difficult or computationally infeasible. This paper is an extension to the author's previous work [14,22]: here we adapt the bi-fidelity SC method [24] to solve the uncertain linear transport equation, and conduct discrete numerical error analysis in all variables (time, space and random uncertainty). The author has studied the Boltzmann equation with random parameters using the bi-fidelity SC method in [22], then in [14] carried out uniform error estimates of the bi-fidelity method for a class of kinetic models, with a discussion of different choices of low-fidelity models.

We mention some relevant work in this research line: there are review articles [27], focusing on some recent progress on employing the multi-fidelity framework to study kinetic UQ problems. Also, the authors in $[12,13,16]$ have constructed multilevel variance reduced Monte Carlo methods for kinetic models-in particular the BGK equations-by adapting the control variate technique in the multilevel Monte Carlo method to reduce the variance. For one-dimensional random variable problem, earlier in [9], the authors have studied the uncertain linear transport 
equation under the diffusive scaling. They use instead the generalized polynomial chaos-based stochastic Galerkin (SG) method, and prove the uniform-in- $\varepsilon$ error estimate and stochastic asymptotic-preserving property.

Here is the outline of the paper. We introduce the linear transport equation with random uncertainties and its diffusion limit in Section 2. In Section 3, we first discuss the high-fidelity solver, which is the Discontinuous Galerkin (DG)-implicit-explicit (IMEX) asymptotic-preserving scheme in the micro-macro decomposition framework developed for the deterministic problem [19]; in subsection 3.2, different ways of choosing low-fidelity models are addressed, either let it be a simplified physics of the diffusion model or the same solver as the high-fidelity while computed on coarser meshes. We study the error analysis for the discrete scheme in Section 4. Numerous numerical experiments are carried out in Section 5 to validate the accuracy and efficiency of our proposed method.

\section{The linear transport equation with random inputs}

In this paper, we consider the linear transport equation in a slab geometry with high-dimensional random inputs:

$$
\begin{aligned}
& \varepsilon \partial_{t} f+v \partial_{x} f=\frac{\sigma(x, z)}{\varepsilon} \mathcal{L}(f), \quad \sigma(x, z) \geq \sigma_{\min }>0, \\
& \mathcal{L}(f)=\frac{1}{2} \int_{-1}^{1} f(t, x, v, z) d v-f(t, x, v, z),
\end{aligned}
$$

where $f(t, x, v, z)$ is the density distribution of particles at time $t \geq 0$, position $x \in \Omega_{x} \subset \mathbb{R}, v=\cos \theta \in \Omega_{v}=[-1,1]$ where $\theta$ is the angle between the moving direction and $x$-axis. Here $\varepsilon$ is the dimensionless Knudsen number, defined by the ratio between particle mean free path and the characteristic length scale, and $\sigma$ is the material cross-section. We consider random inputs coming from the cross-section and initial data. The uncertainty is characterized by the random variable $z \in I_{z} \subset \mathbb{R}^{d}$ with probability density function $\mu(z)$, where $d$ is the dimension of the random variable. The linear operator We define the operator one-dimensional, and the bor boundary conditions. The ir
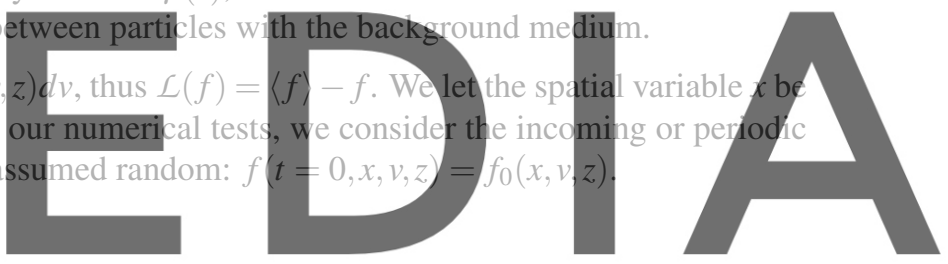

Consider the Hilbert space $L^{2}\left(\Omega_{v} ; d v\right)$ in the velocity space $\Omega_{v}=[-1,1]$, with the.corresponding inner product

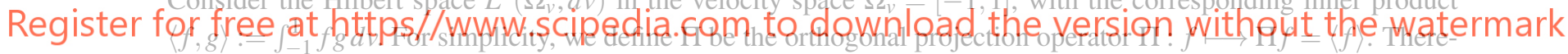
fore, one can write $\mathcal{L}(f)=-(\mathbb{I}-\Pi)(f)$.

We recall the micro-macro decomposition introduced in [20], an approach based on a decomposition of the distribution function into its equilibrium and fluctuation parts. The idea is to apply a projection technique to reformulate the kinetic equation into a coupled system that describes the evolution of both parts. In [19] and [18], DG spatial discretization with arbitrary order accuracy is applied to kinetic transport equations; the authors employ the IMEX strategy in temporal discretization to deal with the stiff terms in the micro-macro system and prove the asymptotic-preserving property of the numerical scheme.

Denote the macroscopic density $\rho:=\Pi f=\langle f\rangle$, then we decompose $f$ into the formulation

$$
f=\langle f\rangle+\varepsilon g=\rho+\varepsilon g .
$$

Here $\rho$ is the equilibrium part of $f$, defined by $\rho=\frac{1}{2} \int_{-1}^{1} f d v$. The fluctuation part $g$ satisfies $\Pi g=\langle g\rangle=0$. Insert the ansatz (2) into (1), apply the operator $\Pi$ and $\mathbf{I}-\Pi$ respectively, we obtain a micro-macro system

$$
\begin{aligned}
& \partial_{t} \rho+\partial_{x}\langle v g\rangle=0, \\
& \partial_{t} g+\frac{1}{\varepsilon}(\mathbf{I}-\Pi)\left(v \partial_{x} g\right)=-\frac{\sigma(z)}{\varepsilon^{2}} g-\frac{1}{\varepsilon^{2}} v \partial_{x} \rho .
\end{aligned}
$$


The diffusion limit For each fixed $z$, in the diffusion limit $\varepsilon \rightarrow 0^{+}$, the system (3)-(4) can be approximated by its leading order of $\varepsilon$ :

$$
\begin{aligned}
& v \partial_{x} \rho+\sigma(x, z) g=0 \\
& \partial_{t} \rho+\partial_{x}\langle v g\rangle=0,
\end{aligned}
$$

which implies a diffusion model [3] given by: $\partial_{t} \rho-\partial_{x}\left(\frac{1}{3 \sigma(x, z)} \partial_{x} \rho\right)=0$.

\section{The bi-fidelity stochastic collocation method}

We refer to [24,33] for a review of the bi-fidelity SC algorithm. In this section, we will discuss the high-fidelity solver and different choices of the low-fidelity models.

\subsection{A fully discretized scheme for the high-fidelity model}

\section{The spatial discretization}

Originated in [28] for neutron transport equations, DG is a class of finite element methods that use completely discontinuous basis functions-usually piecewise polynomials. It has the flexibility that continuous finite element methods do not possess, we refer the readers to $[8,29,30,32]$ for a review.

First, we introduce some notations commonly used [29]. Consider the spatial domain $\Omega_{x}=\left[x_{L}, x_{R}\right]=\bigcup_{i=1}^{N_{x}} I_{i}$, with cell $I_{i}=\left[x_{i-\frac{1}{2}}, x_{i+\frac{1}{2}}\right]$ of length $h_{i}=x_{i+\frac{1}{2}}-x_{i-\frac{1}{2}}$. Note that $x_{L}=x_{\frac{1}{2}}, x_{R}=x_{N_{x}+\frac{1}{2}}$. The discontinuous finite element space is defined as $V_{h}^{\ell}=\left\{u \in L^{2}\left(\Omega_{x}\right):\left.u\right|_{I_{i}} \in P^{\ell}\left(I_{i}\right), \forall i \in\left[1, \cdots, N_{x}\right]\right\}$, where $P^{\ell}(I)$ is the polynomial space with

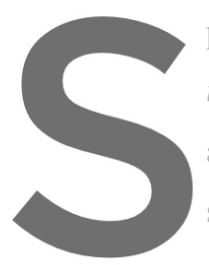
polynomials of degree ut
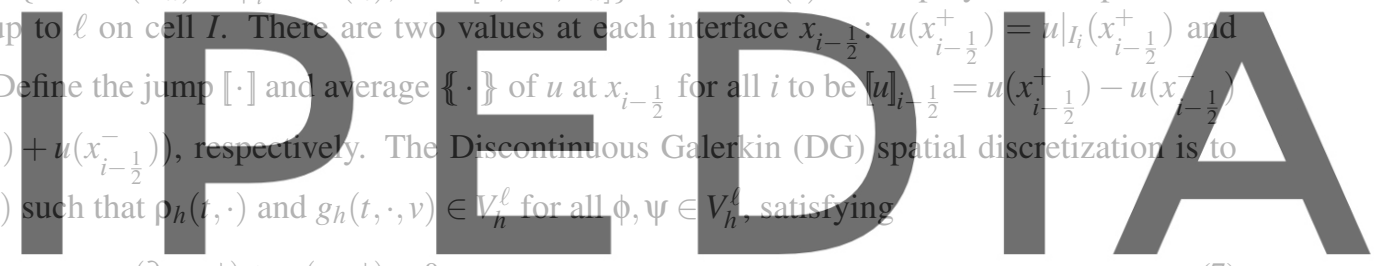

$\left(\partial_{t} \rho_{h}, \phi\right)+a_{h}\left(g_{h}, \phi\right)=0$

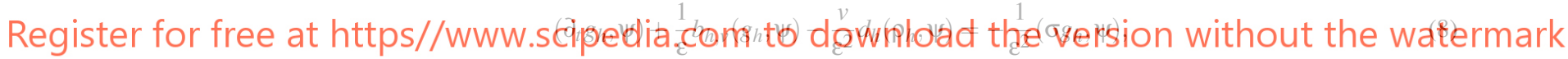
where

$$
\begin{aligned}
& a_{h}\left(g_{h}, \phi\right)=-\sum_{i=1}^{N} \int_{I_{i}}\left\langle v g_{h}\right\rangle \partial_{x} \phi d x-\sum_{i=1}^{N+1} \widehat{\left\langle v g_{h}\right\rangle_{i-\frac{1}{2}}} \llbracket \phi \rrbracket_{i-\frac{1}{2}}, \\
& b_{h, v}\left(g_{h}, \psi\right)=\left(D_{h}\left(g_{h}, v\right)-\left\langle D_{h}\left(g_{h}, v\right)\right\rangle, \psi\right), \\
& d_{h}\left(\rho_{h}, \psi\right)=\sum_{i=1}^{N} \int_{I_{i}} \rho_{h} \partial_{x} \psi d x+\sum_{i=1}^{N+1} \hat{\rho}_{h, i-\frac{1}{2}} \llbracket \psi \rrbracket_{i-\frac{1}{2}} .
\end{aligned}
$$

Here $(\cdot, \cdot)$ denotes the standard inner product for the $L^{2}\left(\Omega_{x} ; d x\right)$ space. The function $D_{h}(\cdot, \cdot)$ is defined based on an upwinding discretization:

$$
\left(D_{h}\left(g_{h}, v\right), \psi\right):=-\sum_{i=1}^{N}\left(\int_{I_{i}} v g_{h} \partial_{x} \psi d x\right)-\sum_{i=1}^{N+1} \widetilde{\left(v g_{h}\right)_{i-\frac{1}{2}}}\left[\psi \rrbracket_{i-\frac{1}{2}}, \quad \psi \in V_{h}^{\ell} .\right.
$$

The upwinding flux is defined by

$$
\widetilde{v g}:=\left\{\begin{array}{l}
v g^{-} \text {if } v>0 \\
v g^{+} \text {if } v<0
\end{array}\right.
$$


We use the left-right alternating flux,

$$
\widehat{\langle v g\rangle}=\langle v g\rangle^{-}, \quad \hat{\rho}=\rho^{+} .
$$

Note that the right-left alternating flux $\left(\widehat{\langle v g\rangle}=\langle v g\rangle^{+}, \hat{\rho}=\rho^{-}\right)$can be analyzed similarly.

The temporal discretization We follow the IMEX strategy and the discretized AP scheme proposed for the telegraph and one-group transport equations in $[18,19]$. Denote the time step size $\Delta t$, and $n$-th time step $t^{n}=n \Delta t$ for all non-negative integer $n$. For fixed $z \in I_{z}$, the discretized numerical scheme used to update $\rho_{h}^{n+1}(x), g_{h}^{n+1}(x, v) \in V_{h}^{\ell}$ at $t^{n+1}$ is shown by

$$
\begin{aligned}
& \left(\frac{\rho_{h}^{n+1}-\rho_{h}^{n}}{\Delta t}, \phi\right)+a_{h}\left(g_{h}^{n}, \phi\right)=0, \\
& \left(\frac{g_{h}^{n+1}-g_{h}^{n}}{\Delta t}, \psi\right)+\frac{1}{\varepsilon} b_{h, v}\left(g_{h}^{n}, \Psi\right)-\frac{v}{\varepsilon^{2}} d_{h}\left(\rho_{h}^{n+1}, \psi\right)=-\frac{1}{\varepsilon^{2}}\left(\sigma g_{h}^{n+1}, \psi\right),
\end{aligned}
$$

for all $\phi, \psi \in V_{h}^{\ell}$, given the approximated solution $\rho_{h}^{n}(x), g_{h}^{n}(x, v) \in V_{h}^{\ell}$ at time $t^{n}$.

\subsection{Low-fidelity models}

In reality, compared to the high-fidelity model-the goal problem, there usually exist less complex low-fidelity models which are employed to capture certain important features of the underlying problem and produce reliable predictions in the random space. There are different ways of choosing the low-fidelity models. We discuss and will numerically implement two choices for the linear transport equation (1).

Choice I: We can adopt the same scheme (14)-(15) as the low-fidelity solver, whereas compute it on a coarser mesh in the physical space, i. Choice III: Rigorous asy One can let the asymptotic conditions, for fixed $\Delta t, h$
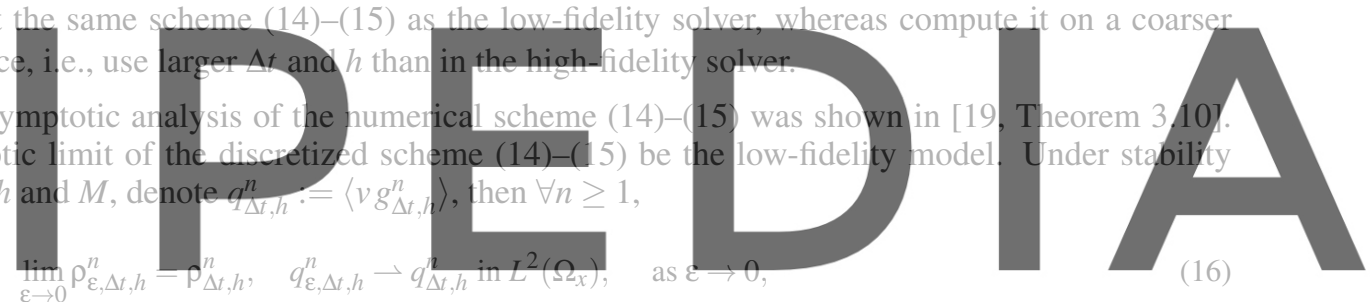

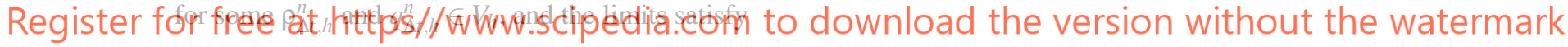

$$
\begin{aligned}
\left(\frac{\left.\rho_{\Delta t, h}^{n+1}-\rho_{\Delta t, h}^{n}, \phi\right)}{\Delta t}\right. & -\sum_{i} \int_{I_{i}} q_{\Delta t, h}^{n} \partial_{x} \phi d x-\sum_{i} \widehat{\left(q_{\Delta t, h}^{n}\right)_{i-\frac{1}{2}}} \| \psi_{\Perp i-\frac{1}{2}}=0, \\
\left(\sigma q_{\Delta t, h}^{n+1}, \psi\right) & =\frac{1}{3}\left(\sum_{i} \int_{I_{i}} \rho_{\Delta t, h}^{n+1} \partial_{x} \psi d x+\sum_{i} \widehat{\left(\rho_{\Delta t, h}^{n+1}\right)_{i-\frac{1}{2}}} \| \psi \rrbracket_{i-\frac{1}{2}}\right),
\end{aligned}
$$

for all $\phi, \psi \in V_{h}^{\ell}$, where the initial data is given by ( $\pi_{h}$ being the projection)

$$
\rho_{\Delta t, h}^{0}=\left.\pi_{h} \rho_{0}\right|_{t=0}, \quad q_{\Delta t, h}^{0}=\left.\pi_{h}\left\langle v g_{0}\right\rangle\right|_{t=0} .
$$

We remark that the limiting scheme (17)-(18) is consistent with the diffusion limit (5)-(6) on the discrete level, in its first order in time form

$$
\partial_{t} \rho+\partial_{x} q=0, \quad \sigma q=-\left\langle v^{2}\right\rangle \partial_{x} \rho=-\frac{1}{3} \partial_{x} \rho
$$

and the local DG spatial discretization [10], noting that $q=\langle v g\rangle$. 


\section{Error analysis at the discretized level}

The error estimate of the DG-IMEX method in its micro-macro decomposition formulation was proved in [19] for the deterministic problem. Let $\rho^{n}, g^{n}$ be the exact solution to the micro-macro system (7)-(8) at time $t^{n}=n \Delta t$, and $\rho_{h}^{n}, g_{h}^{n} \in V_{h}^{\ell}$ be the approximated solutions to the corresponding discretized scheme (14)-(15) at time $t^{n}$. Define the norms

$$
\|\phi\|=\|\phi\|_{L^{2}\left(\Omega_{x}\right)}, \quad\|\| \phi \mid \|=\left(\left\langle\|\phi\|^{2}\right\rangle\right)^{1 / 2} .
$$

We recall the result in [19]. If using the alternating flux (13), then

$$
\begin{aligned}
& \left\|\rho^{n}-\rho_{h}^{n}\right\|^{2}+\varepsilon^{2}\left\|\left|g^{n-1}-g_{h}^{n-1}\right|\right\|^{2} \\
\leq & C\left(\left(1+\varepsilon^{2}\right) h^{2 \ell+2}+\Delta t^{2}+\frac{1}{1-\sigma}\left(\left(1+\varepsilon^{4}\right) \Delta t^{2}+h^{2 \ell+2}+\varepsilon h^{2 \ell+1}\right)\right),
\end{aligned}
$$

under the CFL condition $\Delta t \leq \sigma \Delta t_{\text {stab }}$ and $\Delta t<\frac{1}{2}$, where $\sigma$ is any constant in $(0,1)$.

\subsection{Choice I of the low-fidelity model}

Denote $\rho_{h_{1}}^{H, n}, g_{h_{1}}^{H, n}$ the high-fidelity solutions at $t^{n}$ to the scheme (14)-(15). We consider Choice I in section 3.2, let $\rho_{h_{2}}^{L, n}, g_{h_{2}}^{L, n}$ be the low-fidelity solutions, solved by (14)-(15) on coarser meshes, with $h_{2}>h_{1}$ and $\Delta t_{2}>\Delta t_{1}\left(\Delta t_{2}\right.$ for low; $\Delta t_{1}$ for high).

For simplicity of notation, we define the difference norm between the bi-fidelity and high-fidelity solutions by

$$
\left\|u^{H}-u^{B}\right\|:=\left\|\rho^{H}(z)-\rho^{B}(z)\right\|_{L_{z}^{2}}^{2}+\varepsilon^{2}\|\| g^{H}(z)-g^{B}(z) \mid \|_{L_{z}^{2}}^{2} .
$$
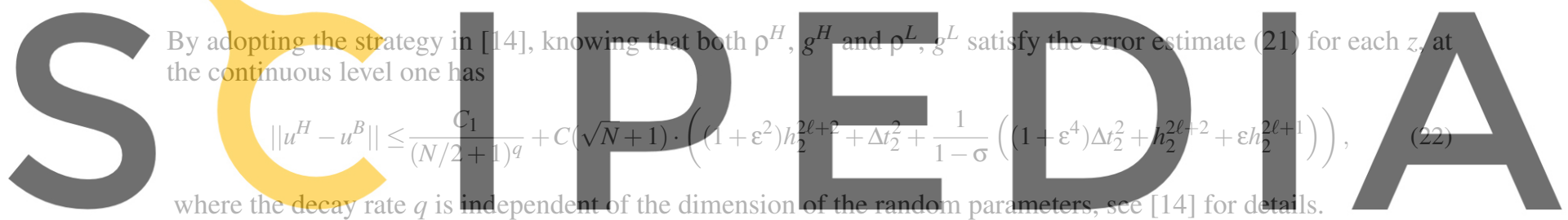

Let us denote the error between the high-fidelity solution with the corresponding discretized numerical solution to

Register fok freevatxhattpsd/www.scipedia.com to download the version without the watermark

$$
\left\|u_{h_{1}}^{H}-u^{H}\right\|:=\left\|\rho_{h_{1}}^{H, n}-\left.\rho^{H, n}\right|_{L_{z}^{2}} ^{2}+\varepsilon^{2}\right\|\left|g_{h_{1}}^{H, n-1}-g^{H, n-1}\right| \|_{L_{z}^{2}}^{2},
$$

of which bound is given in (21), with $h_{1}, \Delta t_{1}$ on the right-hand-side.

Our goal is to estimate the error between the high-fidelity solution and its bi-fidelity approximation at the discretized level, given by

$$
\left\|u_{h_{1}}^{H}-u_{h_{1}}^{B}\right\|:=\left\|\rho_{h_{1}}^{H, n}-\rho_{h_{1}}^{B, n}\right\|_{L_{z}^{2}}^{2}+\varepsilon^{2}\left\|\left|g_{h_{1}}^{H, n-1}-g_{h_{1}}^{B, n-1}\right|\right\|_{L_{z}^{2}}^{2} .
$$

Similar to the error splitting idea introduced in [14], we now have

$$
\begin{aligned}
& \quad\left\|u_{h_{1}}^{H}-u_{h_{1}}^{B}\right\|=\left\|u_{h_{1}}^{H}-\sum_{k=1}^{N} c_{k}(z) u_{h_{1}}^{H}\left(z_{k}\right)\right\|=\left\|u_{h_{1}}^{H}-u^{H}+u^{H}-\sum_{k=1}^{N} c_{k}(z)\left(u_{h_{1}}^{H}\left(z_{k}\right)-u^{H}\left(z_{k}\right)\right)-\sum_{k=1}^{N} c_{k}(z) u^{H}\left(z_{k}\right)\right\| \\
& \leq\left\|u_{h_{1}}^{H}-u^{H}\right\|+\left\|\sum_{k=1}^{N} c_{k}(z)\left(u_{h_{1}}^{H}\left(z_{k}\right)-u^{H}\left(z_{k}\right)\right)\right\|+\left\|u^{H}-\sum_{k=1}^{N} c_{k}(z) u^{H}\left(z_{k}\right)\right\| \\
& \leq\left(C^{\prime} \sqrt{N}+1\right)\left\|u_{h_{1}}^{H}-u^{H}\right\|+\left\|u^{H}-u^{B}\right\|,
\end{aligned}
$$


where in the second term of the above inequality

$$
\begin{aligned}
& \left\|\sum_{k=1}^{N} c_{k}(z)\left(u_{h_{1}}^{H}\left(z_{k}\right)-u^{H}\left(z_{k}\right)\right)\right\| \leq\left(\sum_{k=1}^{N}\left\|c_{k}(z)\right\|^{2}\right)^{1 / 2}\left(\sum_{k=1}^{N}\left\|u_{h_{1}}^{H}\left(z_{k}\right)-u^{H}\left(z_{k}\right)\right\|^{2}\right)^{1 / 2} \\
& \leq \sqrt{N}\left(\sum_{k=1}^{N}\left\|c_{k}(z)\right\|^{2}\right)^{1 / 2} \max _{k}\left\|u_{h_{1}}^{H}\left(z_{k}\right)-u^{H}\left(z_{k}\right)\right\| \leq C^{\prime} \sqrt{N}\left\|u_{h_{1}}^{H}-u^{H}\right\|,
\end{aligned}
$$

we used $\left(\sum_{k=1}^{N}\left\|c_{k}(z)\right\|^{2}\right)^{1 / 2} \sim O(1)$ as in [14].

Based on the splitting in (24), combine (22) and (23), we obtain the error estimate of Choice I and conclude it in the following Theorem:

Theorem 1 The discretized error between the bi-fidelity approximation and high-fidelity solution, where both lowand high-fidelity solvers are the DG-IMEX scheme in the micro-macro decomposition framework (14)-(15), is given by

$$
\leq \frac{C_{1}}{(N / 2+1)^{q}}+C_{2}(\sqrt{N}+1)\left(\left(1+\varepsilon^{2}\right) h_{2}^{2 \ell+2}+\Delta t_{2}^{2}+\frac{1}{1-\sigma}\left(\left(1+\varepsilon^{4}\right) \Delta t_{2}^{2}+h_{2}^{2 \ell+2}+\varepsilon h_{2}^{2 \ell+1}\right)\right),
$$

where $h_{1}, h_{2}$ are the spatial mesh sizes used in high-and low-fidelity solver respectively, the constants $C_{1}, C_{2}$ are independent of $\varepsilon$.

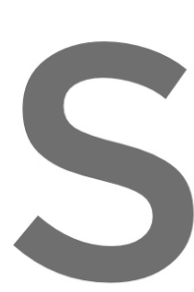

4.2 Choice II of the low-fidelity model

Recall the purely parabolic case in [10, Theorem 2.2], deterministic diffusion mode

S

where the constant $C$ is of order $h$ for even values of $\ell$. For simplicity, we only consider $p$ in the high- and low-

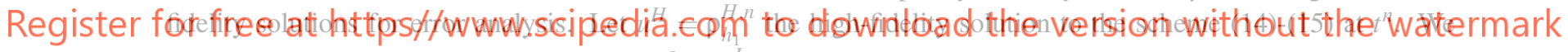

consider Choice II in section 3.2, and let $u^{L}=\rho_{h_{2}}^{L, n}$ solved by the discretized limiting scheme (17)-(18) be the low-fidelity solution. The spatial and temporal sizes used are $h_{1}, \Delta t_{1}$ (for high) and $h_{2}, \Delta t_{2}$ (for low) respectively, assuming $h_{1}<h_{2}$ and $\Delta t_{1}<\Delta t_{2}$.

Denote $\rho_{\varepsilon}$ the exact solution to the micro-macro system (7)-(8), and $\rho_{0}$ the exact solution to the limiting diffusion model (19). The classical diffusion limit theory of linear transport equation $[2,3]$ gives that $\rho_{\varepsilon}$ converges to $\rho_{0}$ as $\varepsilon \rightarrow 0$, for each fixed $z$. Thus at time $t^{n}$, under the $L^{2}\left(\Omega_{x}\right)$ norm defined in (20), we have

$$
\left\|\rho_{\varepsilon}^{n}-\rho_{0}^{n}\right\|^{2} \leq C_{1} \varepsilon^{2}
$$

The estimate (25) and first order temporal discretization of the scheme (17)-(18) indicates that

$$
\left\|\rho_{h_{2}}^{L, n}-\rho_{0}^{n}\right\|^{2} \leq C_{2}\left(h_{2}^{2 \ell}+\left(\Delta t_{2}\right)^{2}\right) .
$$

Now (21) gives us

$$
\left\|\rho_{h_{1}}^{H, n}-\rho_{\varepsilon}^{n}\right\|^{2} \leq C_{3}\left(\left(1+\varepsilon^{2}\right) h_{1}^{2 \ell+2}+\Delta t_{1}^{2}+\frac{1}{1-\sigma}\left(\left(1+\varepsilon^{4}\right) \Delta t_{1}^{2}+h_{1}^{2 \ell+2}+\varepsilon h_{1}^{2 \ell+1}\right)\right) .
$$


Combine (26), (27) and (28), for each $z$ we have

$$
\begin{aligned}
& \left\|\rho_{h_{1}}^{H, n}(z)-\rho_{h_{2}}^{L, n}(z)\right\|^{2} \lesssim\left\|\rho_{h_{1}}^{H, n}(z)-\rho_{\varepsilon}^{n}(z)\right\|^{2}+\left\|\rho_{\varepsilon}^{n}(z)-\rho_{0}^{n}(z)\right\|^{2}+\left\|\rho_{0}^{n}(z)-\rho_{h_{2}}^{L, n}(z)\right\|^{2} \\
\leq & C\left(\left(1+\varepsilon^{2}\right) h_{2}^{2 \ell+2}+\Delta t_{2}^{2}+\frac{1}{1-\sigma}\left(\left(1+\varepsilon^{4}\right) \Delta t_{2}^{2}+h_{2}^{2 \ell+2}+\varepsilon h_{2}^{2 \ell+1}\right)\right)+C^{\prime} \varepsilon^{2},
\end{aligned}
$$

where $C_{1}, C_{2}, C_{3}, C, C^{\prime}$ are all generic constants, and $\sigma$ is any constant in $(0,1)$.

In order to apply the idea of error splitting in [14], we only need the analyticity of low-fidelity solution $\rho_{h_{2}}^{L, n}$, which solves the discretized system (17)-(18). This is obvious, since the solution at time $t^{n+1}$ is a linear combination of the solution at previous time steps. As shown in (17), $\rho_{\Delta t, h}^{n+1}$ depends on $\rho_{\Delta t, h}^{n}, q_{\Delta t, h}^{n}$ and has a linear relation. The initial data $\rho$ and $g$ are assumed analytic in the random space, therefore the analyticity of numerical solution solving (17)-(18) with respect to the random variable is preserved at all time steps.

Employ the framework in [14] and use the estimate (29), we conclude the error analysis of Choice II in the following Theorem:

Theorem 2 The error between the high-fidelity density to the DG-IMEX scheme (14)-(15) and the low-fidelity density to the limiting diffusion model (17)-(18) is given by

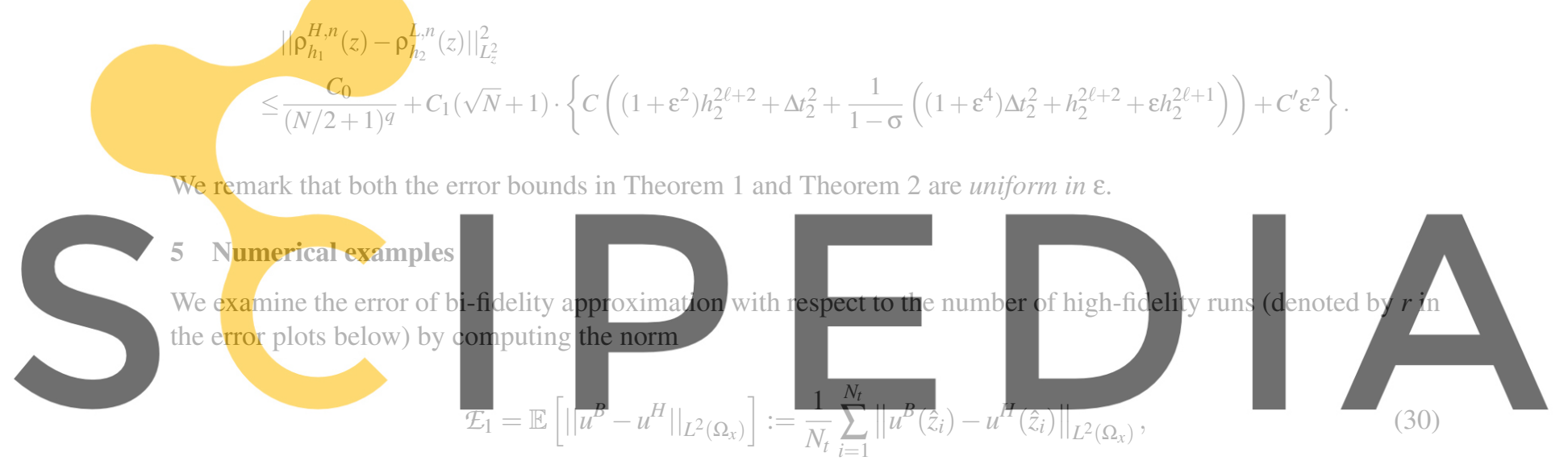

Register for free at https//www.scipedia.com to download the version without the watermark where $u$ represents the macroscopic variable density $\rho$ or flux $q$. For each $\hat{z}_{i}$, the discrete $L^{2}$ norm $\|\cdot\|_{L^{2}(D)}$ in the

physical space $D=\Omega_{x} \times \Omega_{v}$ is evaluated for the difference between $u^{B}$ and $u^{H}$. The test data set $\left\{\hat{z}_{i}\right\}_{i=1}^{N_{t}} \subset I_{z}$ is of size $N_{t}$, randomly chosen and independent of the training set $\Gamma$ of size $M$. This error can be considered as an approximation to the average $L^{2}$ error in the whole space of $D \times I_{z}$.

In the following Problems, for the low-fidelity model, unless particularly specified, we use the same numerical scheme (14)-(15) as the high-fidelity solver, whereas computed on a coarser mesh in the physical space. Let $\ell=2$ in the DG discretization of all tests, and $N_{L}, N_{H}$ be the numbers of spatial discretization points in the low- and high-fidelity models respectively, with $h_{L}=\frac{2 \pi}{N_{L}}, h_{H}=\frac{2 \pi}{N_{H}}$.

\subsection{Problem I: The telegraph model}

We first test the telegraph model [18], where only two velocities $v=1$ and $v=-1$ are allowed. The spatial domain is $x \in[-\pi, \pi]$. The random variables $\left\{z_{k}\right\}_{k=1}^{d}$ are assumed to follow the normal distribution on $\mathbb{R}^{d}$. Consider periodic boundary conditions and a random cross section $\sigma(z)=2\left(1+0.1 \sum_{k=1}^{d} z_{k}^{2}\right)$, where the dimension of the random variable $d=50$. In this case $\langle f\rangle=\frac{1}{2}(f(v=1)+f(v=-1))$, the flux is given by

$$
q:=\langle v g\rangle=\frac{1}{2 \varepsilon}(f(v=1)-f(v=-1)) .
$$


The exact solution can be obtained,

$$
\left\{\begin{array}{l}
\rho(x, t)=\frac{1}{r} \exp (r t) \sin (x), \quad r=\frac{-2}{\sigma(z)+\sqrt{\sigma(z)^{2}-4 \varepsilon^{2}}} \\
q(x, t)=\exp (r t) \cos (x), \\
g(x, v=1, t)=q(x, t) \\
g(x, v=-1, t)=-q(x, t)
\end{array}\right.
$$

Test 1 Consider the diffusive regime with Knudsen number $\varepsilon=10^{-8}$. Here $T=0.01, N_{L}=20, N_{H}=80, M=800$, $N_{t}=2000$. See the solutions and error plots in Figure 1.
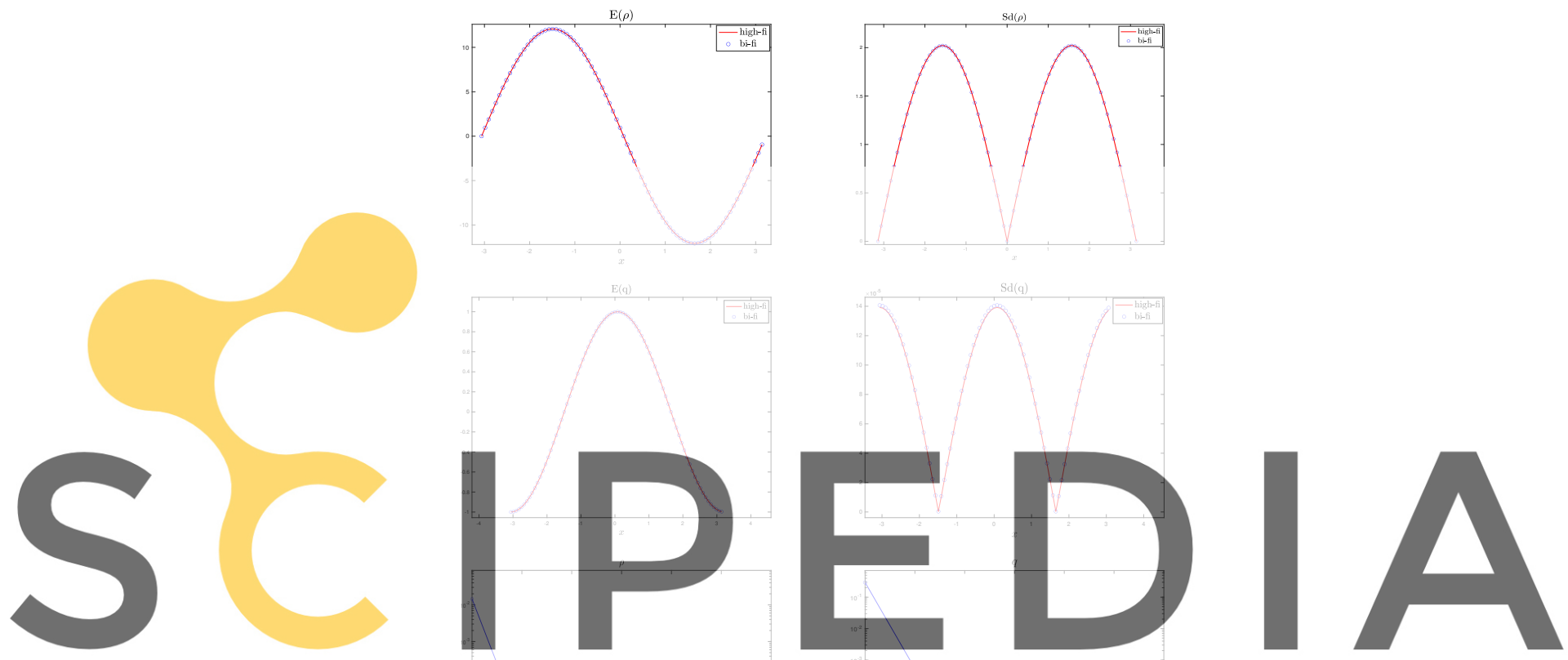

Register for free at https//www.scipedia.com to download the version without the watermark

Figure 1: Test 1. First two rows: Mean and standard deviation of $\rho$ and $q$ for bi-fidelity and high-fidelity solutions by using $r=7$. Third row: Errors of the bi-fidelity approximation for $\rho$ and $q$.

For Test 1 , the mean and standard deviation of the bi-fidelity solutions $\rho$ and $q$ are shown in Figure 1 by adopting the high-fidelity solver only 7 times. We compare them with the high-fidelity solutions and compute the corresponding errors defined by (30), with respect to the number of high-fidelity simulations in the bi-fidelity approximation. One can clearly observe the fast exponential convergence of the errors. Moreover, according to the error convergence plot, we only need to calculate the high-fidelity solver a few times (i.e., $O(1)$ ) on the point set selected by the greedy algorithm.

\subsection{Problem II: Mixed regime}

Instead of discrete velocities $(v=1$ or $v=-1)$ in the telegraph model, we consider continuous velocity $v \in[-1,1]$ in the following numerical examples. Integrals in velocity are computed by the Gauss-Legendre quadrature rule, 
where the quadrature points are chosen as the roots of the Legendre polynomials. Denote Gaussian points used in the low- and high-fidelity models by $q_{L}$ and $q_{H}$ respectively.

We assume the Knudsen number $\varepsilon(x)$ depend on $x$ in a wide range of mixed scalings,

$$
\varepsilon(x)=\varepsilon_{0}+\frac{1}{2}[\tanh (6.5-11 x)+\tanh (11 x-4.5)], \quad x \in[0,1],
$$

which varies smoothly from $\varepsilon_{0}=10^{-5}$ to $O(1)$. Consider the linear transport model (1) with periodic boundary conditions and initial distribution

$$
f_{\text {in }}(x, v)=\frac{\rho_{0}}{2}\left[\exp \left(-\left(\frac{v-0.75}{T_{0}}\right)^{2}\right)+\exp \left(-\left(\frac{v+0.75}{T_{0}}\right)^{2}\right)\right],
$$

where

$$
\rho_{0}(x)=\frac{2+\sin (2 \pi x)}{2}, \quad T_{0}(x)=\frac{5+2 \cos (2 \pi x)}{20} .
$$

The initial condition for the micro-macro system (3)-(4) is computed by

$$
\rho_{0}=\left\langle f_{\text {in }}\right\rangle, \quad g_{0}=\frac{f_{\text {in }}-\rho_{0}}{\varepsilon} .
$$

The random variables $\left\{z_{k}\right\}_{k=1}^{d}$ are assumed to follow the uniform distribution on $I_{z}=[-1,1]^{d}$. We assume the cross-section with random parameters given by
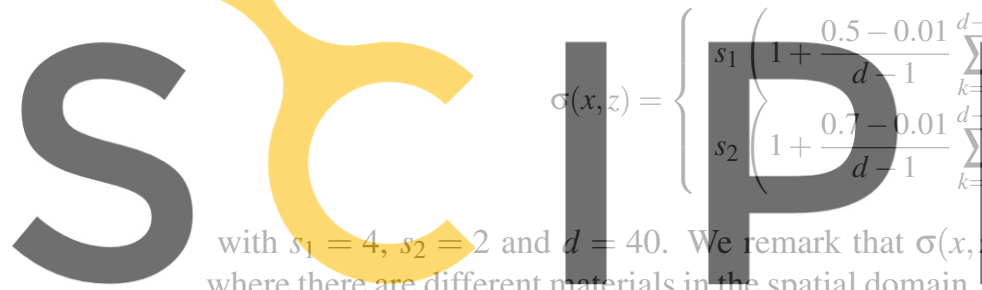

with $s_{1}=4, s_{2}=2$ and

where there are different $n$ rials in spatial domain.
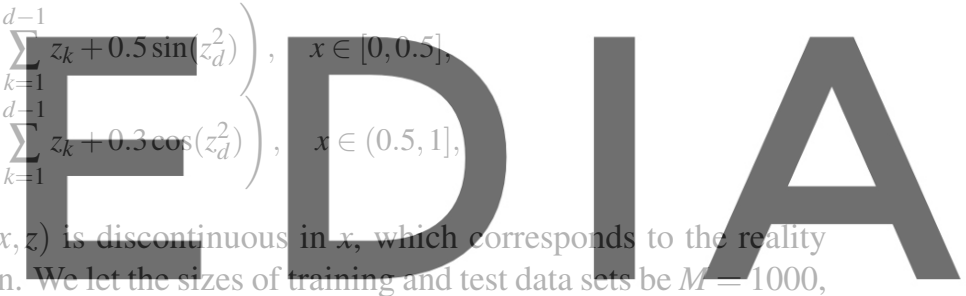

$N_{t}=2000$ respectively. This test is more challenging than Test 1 , since various scales in the model is involved, a

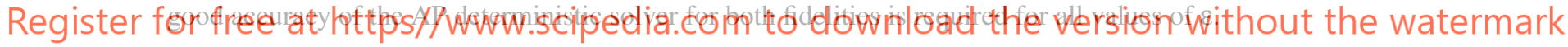

Test 2 In this test, we choose the diffusion limiting scheme (17)-(18) as our low-fidelity solver. Let $T=0.004$, $\Delta t=\frac{1}{10} h^{2}, N_{L}=20, N_{H}=80, q_{H}=16$. See Figure 2 for numerical results. In Figure 2, the mean and standard deviation of $\rho$ and $q$ for bi-fidelity solutions by using $r=10$ are shown. They match really well with the highfidelity solutions. A fast exponential convergence of the errors given by (30) is clearly observed. In particular, with only $r=10$ high-fidelity solver runs, the error of the bi-fidelity method can reach $O\left(10^{-7}\right)$, which is more than satisfactory. The saving of the computational cost in Test 2 is quite remarkable.

\subsection{Problem III: Riemann problem}

In this example, we adopt the limiting scheme (17)-(18), which is consistent with the diffusion limit model (19), as our low-fidelity model. The Riemann problem for the linear transport equation (1) is considered. Assume the random variables $\left\{z_{k}\right\}_{k=1}^{d}$ follow the uniform distribution on $I_{z}=[-1,1]^{d}$.

Let domain $\Omega_{x}=[0,1]$, both boundary conditions

$$
\begin{aligned}
& f(x=0, t, v, z)=1+0.2\left(\frac{z_{1}}{2}+\sum_{k=2}^{d} \frac{z_{k}}{k^{2}}\right), \quad \text { if } v \geq 0, \\
& f(x=1, t, v, z)=0, \quad \text { if } v \leq 0,
\end{aligned}
$$



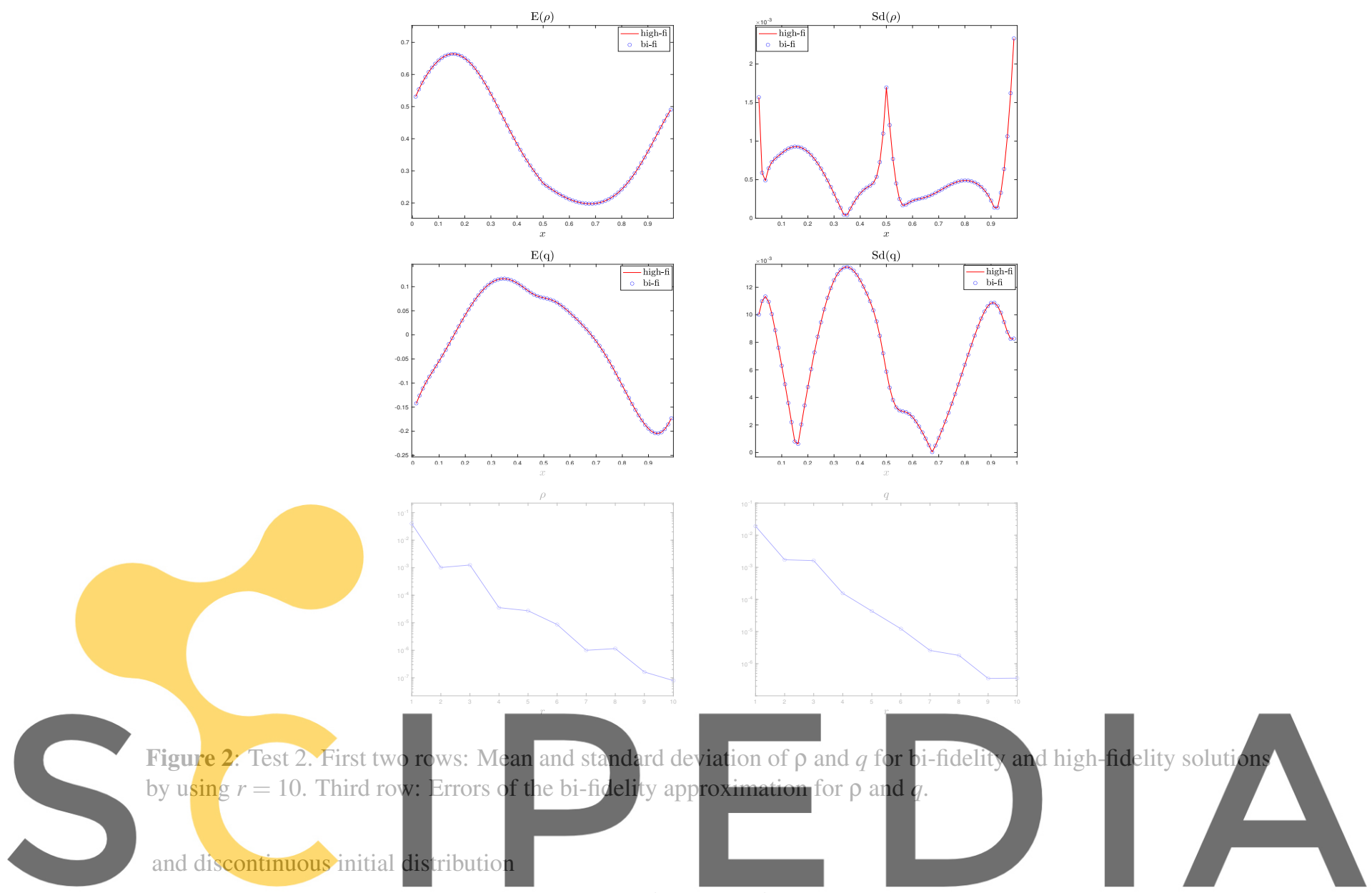

Register for free at https//www.scipedia.com to download the version without the watermark

are uncertain. The uncertain cross-section takes the form: $\sigma(z)=2+4 \sum_{k=2}^{d} \frac{z^{2}}{k}$. In the small mean free path limit, equation (1) leads to the Riemann problem for the heat equation (19) with uncertain initial density

$$
\begin{array}{rr}
\rho(x, t=0, z)=1+0.2\left(\frac{z_{1}}{2}+\sum_{k=2}^{d} \frac{z_{k}}{k^{2}}\right), & 0 \leq x<0.5 ; \\
\rho(x, t=0, z)=0, & 0.5 \leq x \leq 1,
\end{array}
$$

and boundary conditions

$$
\rho(0, t, z)=1+0.2\left(\frac{z_{1}}{2}+\sum_{k=2}^{d} \frac{z_{k}}{k^{2}}\right), \quad \rho(1, t, z)=0 .
$$

As for the flux $q$ in (19), we have the initial condition $q(x, t=0, z)=0$ on $x \in[0,1]$ and the boundary condition $q(0, t, z)=q(1, t, z)=0$.

Test 3 Let $T=0.01, \varepsilon=10^{-4}, d=5, M=200, N_{t}=500, N_{L}=20, N_{H}=80, q_{H}=16, \Delta t=\frac{1}{10} h^{2}$. See Figure 3 for the numerical results. 

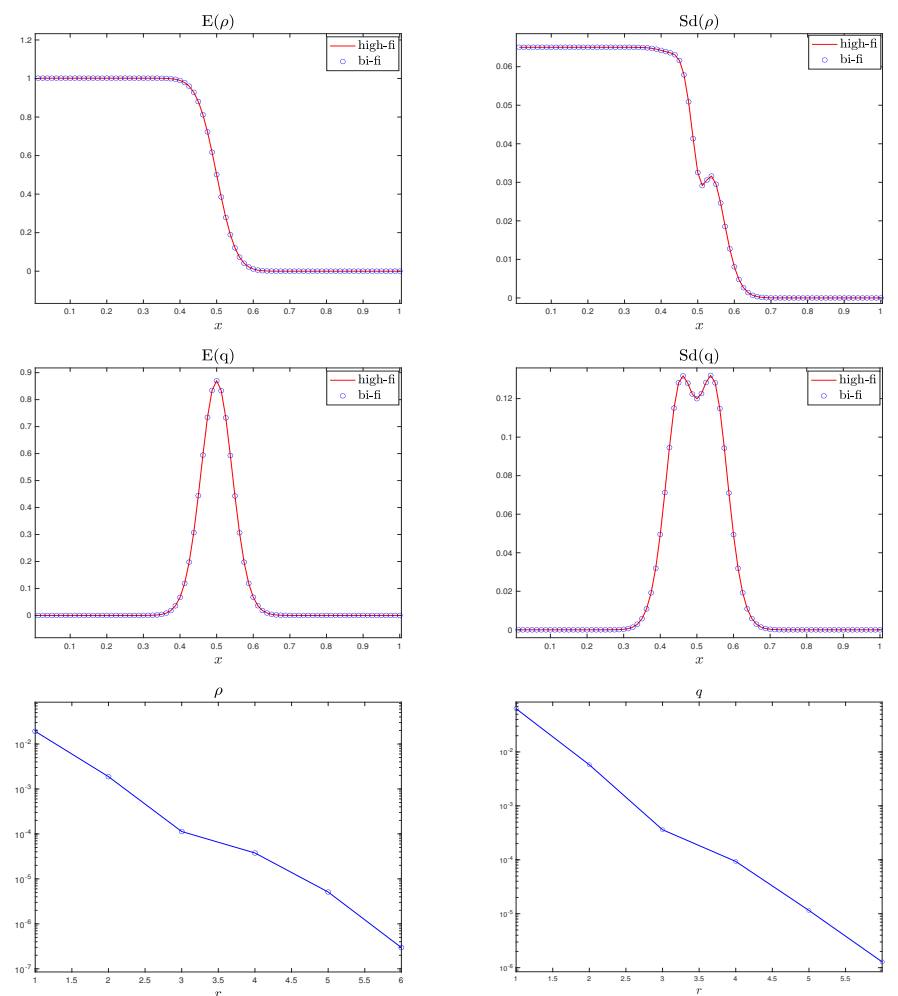

Figure 3: Test 3. First two rows: Mean and standard deviation of $\rho$ and $q$ by using $r=6$. Third row: Errors of the bi-fidelity approximation for $\rho$ and $q$.

From Figure 3 for Test 3, one can again observe a fast exponential convergence of the error for the bi-fidelity approximations, with respect to the number of high-fidelity runs $r$. With only $r=6$, the error reaches down to as small as $O\left(10^{-6}\right)$. This demonstrates the accuracy and efficiency of our bi-fidelity method, in which the limiting scheme (17)-(18) is employed as the low-fidelity model.

\section{Conclusion}

We study a bi-fidelity SC method for the linear transport equation with diffusive scaling and high-dimensional random parameters. Different ways of choosing low-fidelity models, which either describes a simplified physics as the limiting model or is computed on coarser meshes, are explored. We use the framework of bi-fidelity error analysis in [14] for continuous models, based on the analysis tool of hypocoercivity for kinetic equations. One important contribution is that we carefully study the error analysis for the discretized numerical scheme using the DG-IMEX method, and discuss different choices of low-fidelity solvers. The uniform error estimate in the random space are presented. Finally, extensive numerical examples have demonstrated the accuracy and efficiency of our proposed bi-fidelity method for solving the linear transport equation with random inputs.

\section{REFERENCES}

[1] I. BABUŠKA, F. NOBILE, AND R. TEMPONE, A stochastic collocation method for elliptic partial differential equations with random input data, SIAM Rev., 52 (2010), pp. 317-355.

[2] C. Bardos, F. Golse, and D. LeVermore, Fluid dynamic limits of kinetic equations. I. Formal derivations, J. Statist. Phys., 63 (1991), pp. 323-344.

[3] C. Bardos, R. Santos, And R. SEntis, Diffusion approximation and computation of the critical size, Trans. Amer. Math. Soc., 284 
(1984), pp. 617-649.

[4] M. BIERI AND C. Schwab, Sparse high order FEM for elliptic sPDEs, Comput. Methods Appl. Mech. Engrg., 198 (2009), pp. 11491170.

[5] F. Bouchut, F. Golse, And M. Pulvirenti, Kinetic equations and asymptotic theory, Elsevier, 2000.

[6] C. Cercignani, Rarefied Gas Dynamics: From Basic Concepts to Actual Calculations, Cambridge University Press, Cambridge, 2000.

[7] S. CHAPMAN AND T. G. COWLING, The mathematical theory of non-uniform gases. An account of the kinetic theory of viscosity, thermal conduction and diffusion in gases, Third edition, prepared in co-operation with D. Burnett, Cambridge University Press, London, 1970.

[8] Z. CHEN, H. HUANG, AND J. YAN, Third order maximum-principle-satisfying direct discontinuous Galerkin methods for time dependent convection diffusion equations on unstructured triangular meshes, J. Comput. Phys., 308 (2016), pp. 198-217.

[9] Z. CHEN, L. LIU, AND L. MU, DG-IMEX stochastic Galerkin schemes for linear transport equation with random inputs and diffusive scalings, J. Sci. Comput., 73 (2017), pp. 566-592.

[10] B. CocKBURn AND C.-W. Shu, The local discontinuous Galerkin method for time-dependent convection-diffusion systems, SIAM J. Numer. Anal., 35 (1998), pp. 2440-2463.

[11] A. Cohen And R. DeVore, Approximation of high-dimensional parametric PDEs, Acta Numer., 24 (2015), pp. 1-159.

[12] G. Dimarco AND L. PARESChi, Multi-scale control variate methods for uncertainty quantification in kinetic equations, J. Comput. Phys., 388 (2019), pp. 63-89.

[13] - Multiscale variance reduction methods based on multiple control variates for kinetic equations with uncertainties, Multiscale Model. Simul., 18 (2020), pp. 351-382.

[14] I. GAMBA, S. JIN, AND L. LIU, Error estimate of a bi-fidelity method for kinetic equations with random parameters and multiple scales, International Journal for Uncertainty Quantification, to appear (2020).

[15] M. D. GunZburger, C. G. Webster, And G. Zhang, Stochastic finite element methods for partial differential equations with random input data, Acta Numer., 23 (2014), pp. 521-650.

[16] J. HU, L. PARESCHI, AND Y. WANG, Uncertainty quantification for the BGK model of the Boltzmann equation using multilevel variance reduced Monte Carlo methods, preprint, (2020).

[17] J. D. JAKEMAN AND S. G. ROBERTS, Stochastic Galerkin and collocation methods for quantifying uncertainty in differential equations: a review, ANZIAM J., 50 (2008), pp. C815-C830.

[18] J. JANG, F. LI, J. QIU, AND T. XIONG, High order asymptotic preserving DG-IMEX schemes for discrete-velocity kinetic equations in a diffusive scaling, J. Comput. Phys., 281 (2015), pp. 199-224.

[19] J. JANG, F. LI, J.-M. QIU, AND T. XIONG, Analysis of asymptotic preserving DG-IMEX schemes for linear kinetic transport equations in a diffusive scaling, SIAM J. Numer. Anal., 52 (2014), pp. 2048-2072.

[20] M. Lemou AND L. Mieussens, A new asymptotic preserving scheme based on micro-macro formulation for linear kinetic equations in the diffusion limit, SIAM J. Sci. Comput., 31 (2008), pp. 334-368.

[21] Q. LI AND L. WANG, Uniform regularity for linear kinetic equations with random input based on hypocoercivity, SIAM/ASA J. Uncertain. Quantif., 5 (2017), pp. 1193-1219.

[22] L. LiU AND X. ZHU, A bi-fidelity method for the multiscale Boltzmann equation with random parameters, J. Comput. Phys., 402 (2020), pp. 108914, 23.

[23] C. LU AND X. ZHU, Bifidelity data-assisted neural networks in nonintrusive reduced-order modeling, preprint, (2019).

[24] A. Narayan, C. GitTelson, AND D. XiU, A stochastic collocation algorithm with multifidelity models, SIAM J. Sci. Comput., 36 (2014), pp. A495-A521.

[25] A. NaRAYAn AND D. XIU, Stochastic collocation methods on unstructured grids in high dimensions via interpolation, SIAM J. Sci. Comput., 34 (2012), pp. A1729-A1752.

[26] F. Nobile, R. Tempone, And C. G. Webster, A sparse grid stochastic collocation method for partial differential equations with random input data, SIAM J. Numer. Anal., 46 (2008), pp. 2309-2345.

[27] L. PARESCHI, An introduction to uncertainty quantification for kinetic equations and related problems, preprint, (2020).

[28] W. REed AND T. HILL, Triangular mesh methods for the neutron transport equation, Los Alamos Scientific Laboratory, Los Alamos, NM, (1973).

[29] C. SHU, Discontinuous galerkin methods: general approach and stability, numerical solutions of partial differential equations, Advanced courses in Mathematics CRM Barcelona, Birkhäuser, Basel, (2009), pp. 149-201.

[30] C.-W. SHU, High order WENO and DG methods for time-dependent convection-dominated PDEs: a brief survey of several recent developments, J. Comput. Phys., 316 (2016), pp. 598-613.

[31] D. XIU, Efficient collocational approach for parametric uncertainty analysis, Commun. Comput. Phys., 2 (2007), pp. $293-309$.

[32] X. Zhang AND C. SHU, Maximum-principle-satisfying and positivity-preserving high-order schemes for conservation laws: survey and new developments, Proc. R. Soc. Lond. Ser. A Math. Phys. Eng. Sci., 467 (2011), pp. 2752-2776.

[33] X. ZhU, A. NARAYAN, AND D. XIU, Computational aspects of stochastic collocation with multifidelity models, SIAM/ASA J. Uncertain. Quantif., 2 (2014), pp. 444-463. 